\title{
Transformation is adaptation
}

To the Editor - Transformational change is an essential part of society's adaptive and mitigative response to climate change. Existing structures, institutions, habits and priorities need to be critically re-evaluated in light of the risks that climate change poses. Although some transformation will be forced on society as a last resort, some will be more voluntary, positive and anticipatory ${ }^{1,2}$.

The latter sort of adaptation is neglected in the recent Commentary by Dow et al. ${ }^{3}$ on limits to adaptation. Implicitly and explicitly, they present transformational change as outside rather than part of the scope of successful adaptation. Focused on determining where individual or group adaptation limits lie, they present a transformation in behaviour as a response to an 'absence of new adaptation options or resources' and a discontinuity that is 'symptomatic of an adaptation limit being reached'. But rather than a shift from adaptation to non-adaptation, the above represents a move from one major adaptation strategy to another. For example, Dow et al. present a farmer's decision to exit farming as a negative situation of 'abandonment'. But such a move may be highly rational and desirable from a personal, familial and ecological point of view, as examples in Australia suggest ${ }^{1,4,5}$. This is especially the case if it fosters public goods, such as revegetation for carbon sequestration and emissions reductions. Mitigation is a crucial part of successful adaption, and it requires transformational responses.

As is argued about other aspects of climate change ${ }^{6}$, uncertain system dynamics mean that the prediction of adaptation limits - as Dow et al. call for is unfeasible. Adaptation is necessarily experimental. Strategic shifts are frequently going to be needed in response to new conditions and information. They will also emerge as we reassess existing trajectories and goals. Adaptation cannot simply be a conservative project of protecting things we value, at least in the developed world. Our existing values and norms need to be thoroughly re-evaluated as part of the adaptation project.

As others' have pointed out ${ }^{7,8}$, how we frame climate change adaptation strongly influences the way that people approach the issue. It is imperative that the potential for positive transformational change is included in the scope of planned adaptation options and policies.

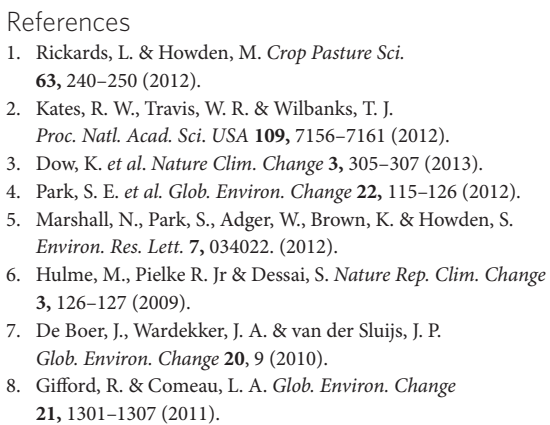

Lauren Rickards

Primary Industries Adaptation Research Network, Melbourne School of Land and Environment and Melbourne Sustainable Society Institute, University of Melbourne, Alice Hoy Building, Parkville, 3010 Melbourne, Victoria 3010, Australia.

e-mail: lauren.rickards@unimelb.edu.au

\section{US maize adaptability}

To the Editor - Butler and Huybers ${ }^{1}$ argue that without adaptation a $2{ }^{\circ} \mathrm{C}$ increase in temperature would decrease maize yields in the US by $14 \%$, but that with adaptation the same temperature increase would decrease yields by only $6 \%$. Butler and Huybers modelled adaptation as decreasing sensitivity to extreme heat (degree days above $29^{\circ} \mathrm{C}$, which they call killing degree days; KDD). Here we show that their conclusions are based on an unjustified interpretation of the data, and that when properly analysed, the data do not support their statement that "losses are almost certainly overestimated if adaptation is not accounted for."

We agree with Butler and Huybers that sensitivity to KDD is, on average, lower in hotter counties. However, this does not necessarily "demonstrate how maize is locally adapted to hot temperatures." It may be that southern counties - because of higher average humidity levels - have a weaker relationship between KDD and vapour pressure deficits (VPD), with high VPD the more direct cause of yield loss ${ }^{2}$. It may also be that southern counties grow shorter-season or hardier varieties, in which case they could be considered adapted, in exchange for lower yields in good conditions. Regardless of the specific reason, it is misleading and unwarranted to simply assume that some counties could benefit from the lower KDD sensitivity observed in other counties.

Consider how the method used by Butler and Huybers would deal with an extreme scenario where yields were exactly zero every year in hot southern counties. This would result in a coefficient of zero on KDD, as well as a zero intercept. By their method, 'adaptation' would apply the zero coefficient on KDD to northern counties as they warm, but preserve the positive intercept in northern counties, suggesting that average yields will remain the same irrespective of any warming. (Supplementary Figure S7 of Butler and Huybers considers only changes in the KDD coefficient, but not the intercept.) A method that fails on this simple example should not, in our view, be trusted.

A more appropriate approach to modelling adaptation would account not only for the change in KDD coefficient in hotter counties, but also for the changes in all other regression parameters. The predicted impacts of a $2{ }^{\circ} \mathrm{C}$ warming is given in Table 1, both for models from Butler and Huybers and a 'costly adaptation 